\title{
A Symbolic Regression Approach to Hepatocellular Carcinoma Diagnosis Using Hypermethylated CpG Islands in Circulating Cell-Free DNA
}

\author{
Rushank Goyal \\ Betsos \\ rgoyal@betsos.org
}

\begin{abstract}
Purpose Hepatocellular carcinoma is the most common primary liver cancer, accounting for $90 \%$ of cases, and a major cause of death worldwide. Despite this, alpha-fetoprotein tests are the only blood-based diagnostic tools available, and their use is limited by their low sensitivity. DNA methylation changes, which have been implicated in a majority of cancers, offer an alternative method of diagnosis through measuring such changes in circulating cell-free DNA present in blood plasma.
\end{abstract}

Method A genetic programming-based symbolic regression approach was applied to gain the benefits of machine learning while avoiding the opacity drawbacks of "black box" models. The data included plasma samples from 36 patients with hepatocellular carcinoma as well as a control group of 55 that contained patients with and without cirrhosis. A 75-25 train-test splitting was done before training.

Results The symbolic regression methodology developed an equation utilizing the methylation levels of three biomarkers, with an accuracy of $91.3 \%$, a sensitivity of $100 \%$, and a specificity of $87.5 \%$ on the test data. The performance matches prior research while providing the added benefits of transparency.

Conclusion Circulating cell-free DNA presents opportunities for minimally invasive early diagnosis of hepatocellular carcinoma, and utilizing transparent machine learning approaches like symbolic regression can allow accurate diagnosis by combining biological and mathematical principles. Future validation of the model obtained here on a larger and more diverse dataset can reveal the potential for such approaches in cancer diagnosis and pave the way for further research.

Keywords Hepatocellular carcinoma, Symbolic regression, Circulating cell-free DNA 


\section{Introduction}

Hepatocellular carcinoma (HCC) is the most common primary liver cancer, with approximately 782,000 new cases and 746,000 deaths yearly (Ferlay et al., 2014). It is the second largest cause of cancer deaths in East Asia and sub-Saharan Africa as well as being the fastest rising cause of cancer-related death in the United States (Rawla et al., 2018). Risk factors such as chronic Hepatitis B (CHB), chronic Hepatitis C (CHC), nonalcoholic steatohepatitis (NASH), and aflatoxin exposure contribute to the development of hepatocelluar carcinoma, with CHB and CHC alone accounting for $75 \%$ of cases (Chen, 2018; Baecker et al., 2018; Ahmed et al., 2019, Wang \& Gribskov, 2019). With alpha-fetoprotein (AFP) tests being the only blood-based diagnostic tool currently available for HCC - the utility of which is hampered by its low sensitivity - there is an unmet need for an effective plasma test to enable early diagnosis (Xu et al., 2017).

CG dinucleotides, also known as CpG pairs, are under-represented in the human genome (21\% of expected) due to the formation of methylcytosine through attachment of methyl groups to cytosine in $\mathrm{CpG}$ pairs; the methylcytosine spontaneously deaminates to thymine (Illingworth \& Bird, 2009). There are, however, interspersions of largely nonmethylated sequences called CpG islands (CGIs) that possess large numbers of GC and CG nucleotides (Deaton \& Bird, 2011). Hypomethylation or hypermethylation of these CGIs acts as a prevalent molecular signature for most cancers, including HCC (Wen et al., 2015). In a number of studies, such methylation changes were detected years before other signs of cancerous development (Shi et al., 2007).

Circulating cell-free DNA (cfDNA) is a term describing extracellular DNA found in body fluids like blood, sputum, urine, etc. (Sun et al., 2019). Its importance and promising outlook as a non-invasive marker for cancer has been recognized, utilizing genetic, methylation, and, to a lesser extent, quantitative analyses (Aarthy et al., 2015; Kustanovich et al., 2019). For HCC in particular, methylation analysis of cfDNA has yielded multiple diagnostic biomarkers, including but not limited to p15, p16, GSTP1 and RASSF1A (Ng et al., 2018).

Symbolic regression is a machine learning (ML) technique that attempts to create a mathematical expression to explain the target variable utilizing a range of basic mathematical functions, and, specifically, genetic programming-based symbolic regression (GPSR) is an implementation of SR that uses genetic programming to search the massive SR solution spaces effectively (Wilstrup \& Kasak, 2021). GPSR has been able to perform well in deriving expressions for real-world applications in fields as disparate as biology, robotics, physics and finance (Orzechowski et al. 2018). Evidence suggests that it outperforms other ML techniques on smaller datasets, which is useful in clinical and biological contexts where data is often limited (Wilstrup \& Kasak, 2021). Its transparency compared to other "black box" machine learning models also makes it more suited to application in clinical settings (Quinn et al. 2021; Price, 2018). 


\section{Materials and Methods}

The data used for the analysis were obtained from the NCBI Gene Expression Omnibus with the accession number GSE63775. The data consist of plasma samples from 55 control subjects, out of which 17 had cirrhosis, and 36 HCC patients (Wen et al., 2015). Values for each CGI in the samples are measured in methylated alleles per million mapped reads (MePM). Stratified random train-test splitting was performed in a 75-25 ratio (random seed of 1) to obtain the training and testing sets respectively.

Python 3.7.12 was used, with pandas and numpy for preprocessing, matplotlib and seaborn for plotting and visualization, scikit-learn for train-test splitting and model evaluation, and gplearn for training the symbolic classifier model. A symbolic classifier works by first developing a symbolic regressor and then passing the output through a logistic function to produce a prediction value, which corresponds to 0 (negative) if less than 0.5 , and 1 (positive) otherwise. The symbolic classifier was trained using population_size $=2000$ and parsimony_coefficient $=0.01$, with a random seed of 1 to ensure reproducibility Andrew L. Beam, 2020).

\section{Results}

Figure 1 shows the output expression in a tree form. Equation 1 shows the final model as a standard mathematical equation, where $c h r_{5}, c h r_{21}$ and $c h r_{19}$ represent the levels of the three CGIs identified as biomarkers by GPSR - chr5:92923487-92924497, chr21:40757602-40757900 and chr19:41531804-41532051, respectively. $x$ is the output, and can be put through the logistic function to obtain the probability $(p)$ of hepatocellular carcinoma, as shown in Equation 2. A probability less than 0.5 indicates that the absence of HCC is more likely, and vice versa.

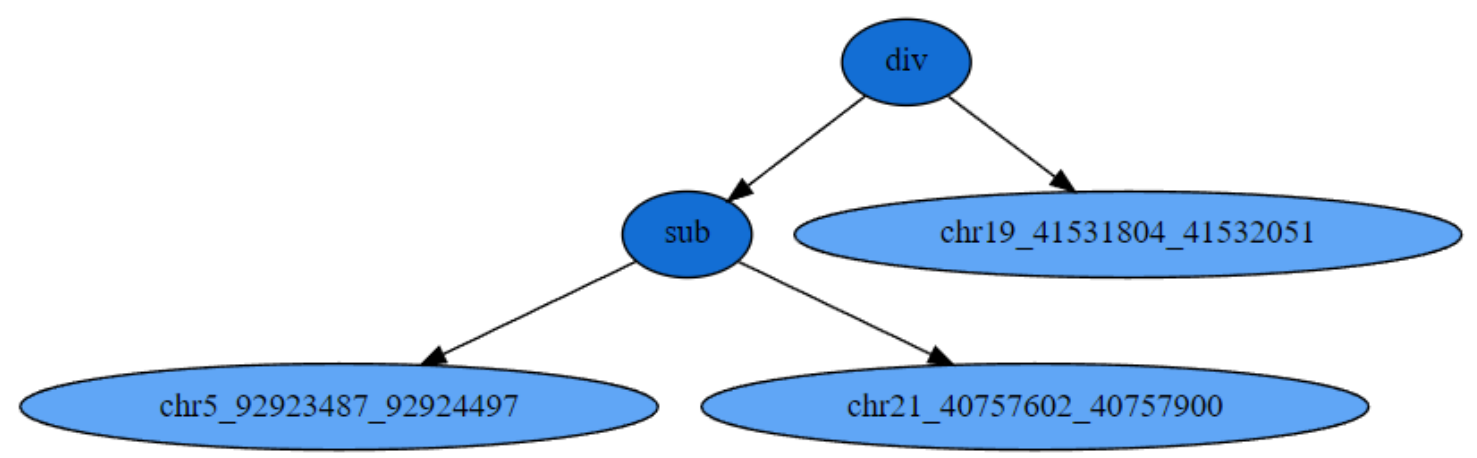

Figure 1: Output Equation as Tree

$$
x=\frac{c h r_{5}-c h r_{21}}{c h r_{19}}
$$




$$
p=\frac{1}{1+e^{-x}}
$$

The results when utilizing this equation for classification on the testing dataset are shown in Figure 2 in the form of a confusion matrix. The accuracy comes out to be $91.3 \%$, and the sensitivity and specificity are $100 \%$ and $87.5 \%$, respectively.

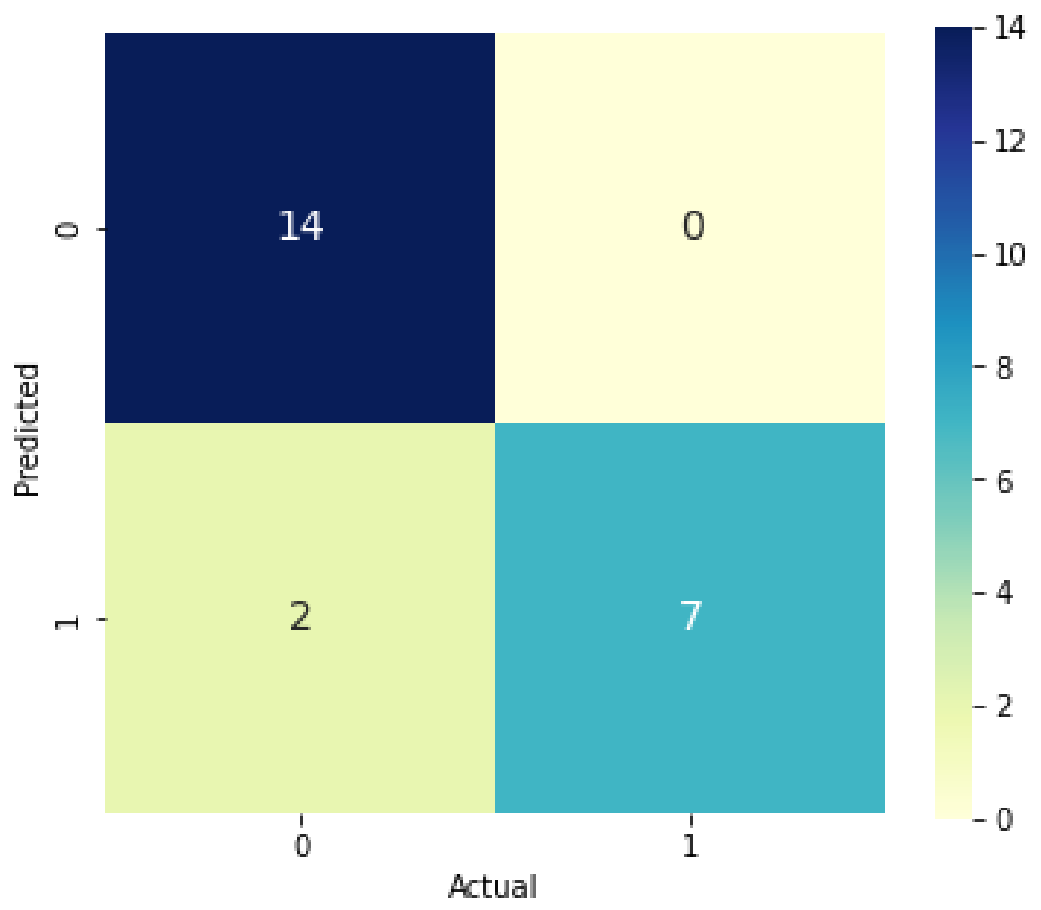

Figure 2: Confusion Matrix on Test Data

\section{Discussion}

Prior ML work in this area has attained similar performances, though it lacks the explainability of symbolic regression (Filho et al., 2020, Khalid et al., 2015). Utilizing a combination of random forest, the least absolute shrinkage and selection operator (LASSO) and logistic regression, $\mathrm{Xu}$ et al. (2017) obtained a sensitivity of $83.3 \%$ and a specificity of $90.5 \%$ on their validation set. Zhang et al. (2020) were able to get a sensitivity and specificity of $91.93 \%$ and $100 \%$ respectively using 11 biomarkers identified by a support vector machine (SVM) model. Another study also used an SVM to achieve a precision (positive predictive value) of $96 \%$ and a recall (sensitivity) of $86 \%$ (Gonçalves et al., 2021).

Multiple studies have identified blood plasma biomarkers without applying ML. Lin et al. (2005) showed that around $77 \%$ of HCC patients possess p16 methylation and $41 \%$ possess DAPK methylation. $55.7 \%$ of patients have a methylated CCND2 gene (Tsutsui et al., 2010). RASSF1A hypermethylation was present in $42.5 \%$ of patients, correlating with tumor size (Yeo et al., 2005). Ji et al. (2004) identified MT1M and MT1G as biomarkers with high 
medRxiv preprint doi: https://doi.org/10.1101/2022.01.25.22269799; this version posted February 14, 2022. The copyright holder for this preprint (which was not certified by peer review) is the author/funder, who has granted medRxiv a license to display the preprint in perpetuity. All rights reserved. No reuse allowed without permission.

Hepatocellular Carcinoma Diagnosis Using cfDNA

specificities of $93.5 \%$ and $87.1 \%$ respectively, though their sensitivities were low, at $48.8 \%$ and $70.2 \%$ respectively.

Compared to simple biomarker analysis, machine learning techniques display better performance due to their more complex nature. The symbolic regression approach described in this paper, however, attains similar sensitivity and specificity to conventional ML approaches, is much more transparent and allows for mathematical reasoning of the results in a biological context, which can be a source of useful further research (Narayanan et al., 2022, Cardoso et al. 2020).

Since the data in this study contained cfDNA - which can be obtained from blood plasma - rather than analyzing liver tissue, the results present possibilities for minimally invasive diagnosis of HCC. Indeed, cfDNA holds potential for improved early cancer diagnosis in general (Stewart et al., 2018). Further analysis of the results on a larger and more diverse validation set as well as biological analyses of the three-biomarker signature are important, but the results obtained here show initial promise.

\section{Data Availability Statement}

The data are available in the National Center for Biotechnology Information's Gene Expression Omnibus (NCBI GEO) under the accession number GSE63775.

\section{Declaration of Conflicts of Interest}

No funding was received for conducting this study. The author has no relevant financial or non-financial interests to disclose.

\section{Ethical Declarations}

This research study was conducted retrospectively using human subject data made available by prior research. Ethical approval was not required, as confirmed by the institutional review board (IRB) of the Sri Aurobindo Institute of Medical Sciences in Indore, India.

\section{References}

Aarthy, R., Mani, S., Velusami, S., Sundarsingh, S., \& Rajkumar, T. (2015, Dec). Role of Circulating Cell-Free DNA in Cancers. Molecular Diagnosis 83 Therapy, 19(6), 339-350. doi: doi:10.1007/s40291-015-0167-y

Ahmed, O., Liu, L., Gayed, A., Baadh, A., Patel, M., Tasse, J., ... Arslan, B. (2019, Jan). The Changing Face of Hepatocellular Carcinoma: Forecasting Prevalence of Nonalcoholic Steatohepatitis and Hepatitis C Cirrhosis. Journal of Clinical and Experimental Hepatology, 9(1), 50-55. doi: doi: $10.1016 /$ j.jceh.2018.02.006

Andrew L. Beam, P. (2020, Jan). Challenges to the Reproducibility of Machine Learning Models in Health Care. JAMA, 323(4), 305-306. doi: doi:10.1001/jama.2019.20866

Baecker, A., Liu, X., La Vecchia, C., \& Zhang, Z.-F. (2018, May). Worldwide Incident Hepatocellular Carcinoma Cases Attributable to Major Risk Factors. European journal of cancer prevention 
: the official journal of the European Cancer Prevention Organisation (ECP), 27(3), 205. doi: doi: 10.1097/CEJ.0000000000000428

Cardoso, P., Branco, V. V., Borges, P. A. V., Carvalho, J. C., Rigal, F., Gabriel, R., ... Correia, L. (2020). Automated Discovery of Relationships, Models, and Principles in Ecology. Frontiers in Ecology and Evolution, 0. doi: doi:10.3389/fevo.2020.530135

Chen, C.-J. (2018, Apr). Global elimination of viral hepatitis and hepatocellular carcinoma: opportunities and challenges. Gut, 67(4), 595-598. doi: doi:10.1136/gutjnl-2017-315407

Deaton, A. M., \& Bird, A. (2011, May). CpG islands and the regulation of transcription. Genes $\&$ Development, 25(10), 1010-1022. doi: doi:10.1101/gad.2037511

Ferlay, J., Soerjomataram, I., Dikshit, R., Eser, S., Mathers, C., Rebelo, M., ... Bray, F. (2014, Oct). Cancer incidence and mortality worldwide: Sources, methods and major patterns in GLOBOCAN 2012. International Journal of Cancer, 136 (5), E359-E386. doi: doi 10.1002/ijc.29210

Filho, R. M., Lacerda, A., \& Pappa, G. L. (2020, Jul). Explaining Symbolic Regression Predictions. In 2020 IEEE Congress on Evolutionary Computation (CEC) (pp. 1-8). IEEE. doi: doi:10.1109/CEC48606.2020.9185683

Gonçalves, E., Reis, M., Pereira-Leal, J. B., \& Cardoso, J. (2021, Jun). DNA methylation fingerprint for the diagnosis and monitoring of hepatocellular carcinoma from tissue and liquid biopsies. medRxiv, 2021.06.01.21258144. Retrieved from https://doi.org/10.1101/2021.06.01.21258144

Illingworth, R. S., \& Bird, A. P. (2009, Jun). CpG islands - 'A rough guide'. FEBS Letters, 583(11), 1713-1720. doi: doi:10.1016/j.febslet.2009.04.012

Ji, X.-F., Fan, Y.-C., Gao, S., Yang, Y., Zhang, J.-J., \& Wang, K. (2014, Apr). MT1M and MT1G promoter methylation as biomarkers for hepatocellular carcinoma. World Journal of Gastroenterology : WJG, 20 (16), 4723. doi: doi:10.3748/wjg.v20.i16.4723

Khalid, M. H., Tuszynski, P. K., Szlek, J., Jachowicz, R., \& Mendyk, A. (2015, Dec). From Black-Box to Transparent Computational Intelligence Models: A Pharmaceutical Case Study. In 2015 13th International Conference on Frontiers of Information Technology (FIT) (pp. 114-118). IEEE. doi: doi:10.1109/FIT.2015.30

Kustanovich, A., Schwartz, R., Peretz, T., \& Grinshpun, A. (2019, Aug). Life and death of circulating cell-free DNA. Cancer Biology \& Therapy, 20(8), 1057-1067. doi: doi:10.1080/15384047.2019.1598759

Lin, Q., Chen, L.-b., Tang, Y.-m., \& Wang, J. (2005, Dec). Promoter hypermethylation of p16 gene and DAPK gene in sera from hepatocellular carcinoma (HCC) patients. Chinese Journal of Cancer Research, 17(4), 250-254. doi: doi $10.1007 / \mathrm{s} 11670-005-0020-7$

Narayanan, H., Cruz Bournazou, M. N., Guillén Gosálbez, G., \& Butté, A. (2022, Feb). Functional-Hybrid modeling through automated adaptive symbolic regression for interpretable mathematical expressions. Chemical Engineering Journal, 430, 133032. doi: doi:10.1016/j.cej.2021.133032

Ng, C. K. Y., Di Costanzo, G. G., Terracciano, L. M., \& Piscuoglio, S. (2018). Circulating Cell-Free DNA in Hepatocellular Carcinoma: Current Insights and Outlook. Frontiers in Medicine, 0. doi: doi:10.3389/fmed.2018.00078

Orzechowski, P., La Cava, W., \& Moore, J. H. (2018, Jul). Where are we now? a large benchmark study of recent symbolic regression methods. In GECCO '18: Proceedings of the Genetic and Evolutionary Computation Conference (pp. 1183-1190). New York, NY, USA: Association for Computing Machinery. doi: doi: $10.1145 / 3205455.3205539$

Price, W. N. (2018, Dec). Big data and black-box medical algorithms. Science Translational Medicine, 10(471), eaao5333. doi: doi $10.1126 /$ scitranslmed.aao5333

Quinn, T. P., Jacobs, S., Senadeera, M., Le, V., \& Coghlan, S. (2021, Aug). The three ghosts of medical AI: Can the black-box present deliver? Artificial Intelligence in Medicine, 102158.. doi: doi:10.1016/j.artmed.2021.102158

Rawla, P., Sunkara, T., Muralidharan, P., \& Raj, J. P. (2018). Update in global trends and aetiology of hepatocellular carcinoma. Contemporary Oncology, 22(3), 141. doi: doi:10.5114/wo.2018.78941

Shi, H., Wang, M. X., \& Caldwell, C. W. (2007, Sep). CpG islands: their potential as biomarkers for cancer. Expert Review of Molecular Diagnostics, 7(5), 519-531. doi: doi:10.1586/14737159.7.5.519

Stewart, C. M., Kothari, P. D., Mouliere, F., Mair, R., Somnay, S., Benayed, R., ... Tsui, D. W. Y. (2018, Apr). The value of cell-free DNA for molecular pathology. Journal of pathology, 244(5), 616. doi: doi: $10.1002 /$ path.5048

Sun, Y., An, K., \& Yang, C. (2019, Jul). Circulating Cell-Free DNA. In Liquid Biopsy. IntechOpen. doi: doi: 10.5772 /intechopen. 80730

Tsutsui, M., Iizuka, N., Moribe, T., Miura, T., Kimura, N., Tamatsukuri, S., ... Oka, M. (2010, Apr). Methylated cyclin D2 gene circulating in the blood as a prognosis predictor of hepatocellular carcinoma. Clinica Chimica Acta, 411(7), 516-520. doi: doi:10.1016/j.cca.2010.01.004 
Wang, S., \& Gribskov, M. (2019, Jun). Transcriptome analysis identifies metallothionein as biomarkers to predict recurrence in hepatocellular cacinoma. Molecular Genetics 83 Genomic Medicine, 7(6), e693. doi: doi:10.1002/mgg3.693

Wen, L., Li, J., Guo, H., Liu, X., Zheng, S., Zhang, D., ... Peng, J. (2015, Nov). Genome-scale detection of hypermethylated CpG islands in circulating cell-free DNA of hepatocellular carcinoma patients - Cell Research. Cell Research, 25, 1250-1264. doi: doi:10.1038/cr.2015.126

Wilstrup, C., \& Kasak, J. (2021, Mar). Symbolic regression outperforms other models for small data sets. ArXiv e-prints. Retrieved from https://arxiv.org/abs/2103.15147v3

Xu, R.-h., Wei, W., Krawczyk, M., Wang, W., Luo, H., Flagg, K., .. Zhang, K. (2017, Nov). Circulating tumour DNA methylation markers for diagnosis and prognosis of hepatocellular carcinoma - Nature Materials. Nature Materials, 16, 1155-1161. doi: doi:10.1038/nmat4997

Yeo, W., Wong, N., Wong, W.-L., Lai, P. B. S., Zhong, S., \& Johnson, P. J. (2005, Apr). High frequency of promoter hypermethylation of RASSF1A in tumor and plasma of patients with hepatocellular carcinoma. Liver International, 25(2), 266-272. doi: doi:10.1111/j.1478-3231.2005.01084.x

Zhang, Z.-M., Tan, J.-X., Wang, F., Dao, F.-Y., Zhang, Z.-Y., \& Lin, H. (2020). Early Diagnosis of Hepatocellular Carcinoma Using Machine Learning Method. Frontiers in Bioengineering and Biotechnology, 0. doi: doi: $10.3389 /$ fbioe.2020.00254 